\title{
SALUD
}

\section{Vigilancia de infecciones del torrente sanguíneo relacionadas a dispositivos de acceso venoso central en una unidad de cuidados intensivos, años 2015-2017}

\author{
Zunilda Garay Duarte ${ }^{1}$
}

\begin{abstract}
Resumen
Introducción: Las infecciones del torrente sanguíneo asociadas con los catéteres venosos centrales (ITS/CVC) son un problema de salud pública, debido a la frecuencia con que se producen, la morbilidad y mortalidad que provocan y la carga que imponen a los pacientes, al personal sanitario y a los sistemas de salud y por ser procesos clínicos potencialmente evitables, sobre todo en países en vías de desarrollo que presentan deficiencias en los servicios de salud. El uso de los datos de vigilancia del propio hospital ha mostrado tener un efecto beneficioso en la sensibilización del personal sanitario en relación con las prácticas de prevención y control de infecciones.
\end{abstract}

Objetivo: Determinar la incidencia de infecciones del torrente sanguíneo relacionadas a dispositivos de acceso venoso central en una Unidad de Cuidados Intensivos.

Material y Método: Estudio observacional, descriptivo, retrospectivo. Fueron incluidos todos los pacientes con infecciones relacionados a CVC de la Unidad de Cuidados Intensivos Adultos (UCIA) del Hospital de tercer nivel de complejidad, durante los años 2015 - 2017. Los datos se obtuvieron de la vigilancia activa realizada en la UCIA. Se incluyeron todos los pacientes ingresados a la UCIA, que presentaron ITS asociadas a CVC durante el periodo de estudio.

Definición de Caso: ITS asociada a vía central: Es la ITS primaria en portadores de una vía o catéter central en el momento de la detección o durante las 48 horas anteriores a la aparición de la infección. Variables utilizadas: días de uso de dispositivos invasivos, tasa de infección del torrente sanguíneo asociado a catéter venoso central reportado, microorganismos aislados. Cálculo de la tasa: La tasa de infección del torrente sanguíneo por 1.000 días-vía central. En relación a los microorganismos aislados, los resultados estadísticos fueron procesados por el método porcentual.

Resultados: La tasa de ITS/CVC por 1000 días de exposición al dispositivo fue del 9,2 en el año 2015, 9 en el año 2016 y 6,6 en el año 2017. Fueron aislados 86 microorganismos, siendo los microorganismos más frecuente Staphylococcus

1 Universidad Nacional de Asunción, Facultad de Ciencias Médicas, Hospital de Clínicas.

E-mail: zunichamorro82@hotmail.com

DOI: $10.26885 /$ rcei.foro.2018.130 


\section{Vigilancia de infecciones del torrente sanguíneo. Garay Duarte}

41 (48\%) de los cuales correspondió al Staphylococcus haemolitycus 6(14\%) Staphylococcus epidermidis 12(12\%) SCN 8(8\%) Staphylococcus hominis 9 (9\%) Staphylococcus aureus 6 (6\%) Acinetobacter baumanii complex 10(12\%) klebsiella pneumoniae 11(13\%) Enterococcus 12(14\%) de los cuales fueron E. faecalis 6(6\%) Enterococcus faecium 4 (4\%) Enterococcus sp 2(2\%) E. cloacae $4(5 \%)$ Escherichia coli 2 (2\%) Pseudomonas eruginosa 2(2\%) y Cándida sp $1(1 \%)$, de los cuales el 73\% (30/41) Staphylococcus presentaron resistencia a la meticilina, el $70 \%(7 / 10)$ de Acinetobacter fueron multirresistentes por impemeabilidad, el 20\% (2/10) fueron Productoras de Carbapenemasas, el $50 \%(5 / 11)$ de las K. pneumoniae fueron mutirresistentes (productores de Betalactamasa de espectro extendido (BLEE)con resistencia a las cefalosporina, el 20\% (2/11) resultaron Probables Productoras de Carbapenemasa, el 58\% (7/12) de los Enterococcus presentaron resistencia a la Vancomicina, el 50\% (2/4) de Enterobacter y Escherichia coli fueron productores de Betalactamasa de espectro extendido (BLEE), el 50\%(1/2) de Pseudomonas con multirresistencia por impermeabilidad La implementación de estrategias multimodales como los cuidados desde el proceso de inserción y de mantenimiento del acceso venoso central, así la educación, compromiso del equipo, cultura de seguridad y procesos de vigilancia tienen impacto en la disminución de las tasas de ITS/CVC.

Conclusiones: El estudio demostró una disminución de la tasa de ITS/CVC y la frecuencia de utilización de catéteres venosos centrales.

Palabras clave: infecciones del torrente sanguíneo, dispositivos invasivos.

\section{RefERENCiAs}

Londoño F., Á. L., Ardila, F., M. \& Ossa, P. D. (2011). Epidemiología de la infección asociada a catéter venoso central. Revista Chilena De Pediatría, 82(6), 493-501.

Ferrer, B. (2014) Infecciones relacionadas con el uso de los catéteres vasculares. Enferm Infecc Microbiol Clin., 32(2), p. 115-124.

Teresa, V. \& Alberto, F. (2015). Estudio de costo de las infecciones del torrente sanguíneo asociadas a catéter vascular central en pacientes adultos en Chile / Cost evaluation of catheter-related bloodstream infections in adult patients in Chile. Revista Chilena De Infectología, (6), p. 634.

Smilja, K. (2011). International Federational of Infection, IFIC. Conceptos básicos de control de infecciones de IFIC (2a ed.). Washington, Estados Unidos: International Federation of Infection Control.

Organización Mundial de la Salud. (2012). Vigilancia Epidemiológica de las infecciones asociadas a la atención de la salud. Información para Gerentes y Directivos (3 ${ }^{\text {a ed.). }}$. Washington, Estados Unidos: OPS. 